\title{
Fused Image
}

National Cancer Institute

\section{Source}

National Cancer Institute. Fused Image. NCI Thesaurus. Code C94904.

Results of two imaging modalities after a registration process is used to merge these.

Their images can be displayed with different colors as if one were on top of the other. 